\title{
Éclairages bibliographiques
}

Jean-Claude Sergeant

\section{OpenEdition}

\section{Journals}

Édition électronique

URL : http://journals.openedition.org/rfcb/719

DOI : $10.4000 /$ rfcb.719

ISSN : 2429-4373

Éditeur

CRECIB - Centre de recherche et d'études en civilisation britannique

Édition imprimée

Date de publication : 1 février 2002

Pagination : 95-98

ISBN : 2-911580-13-3

ISSN : 0248-9015

Référence électronique

Jean-Claude Sergeant, «Éclairages bibliographiques », Revue Française de Civilisation Britannique [En ligne], XI-3 | 2002, mis en ligne le 21 mars 2016, consulté le 01 mai 2019. URL : http:// journals.openedition.org/rfcb/719; DOI : 10.4000/rfcb.719

Ce document a été généré automatiquement le 1 mai 2019.

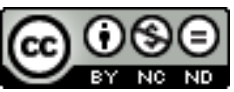

Revue française de civilisation britannique est mis à disposition selon les termes de la licence Creative Commons Attribution - Pas d'Utilisation Commerciale - Pas de Modification 4.0 International. 


\title{
Éclairages bibliographiques
}

\author{
Jean-Claude Sergeant
}

1 La fin de la première mandature de Tony Blair en tant que premier ministre a suscité une activité éditoriale pléthorique. L'intérêt des observateurs était à la mesure de la portée des nouvelles politiques mises en œuvre et reflétait l'attrait qu'exerce sur les spécialistes l'ambiguité de l'œuvre réformatrice de Tony Blair qui échappe, dans une large mesure, aux catégories traditionnelles de l'analyse segmentée par les démarcations idéologiques.

2 Par souci de commodité, on a regroupé l'essentiel de la production éditoriale sur le sujet en deux ensembles distincts en fonction de leur lieu de publication. Cet inventaire ne prétend nullement à l'exhaustivité, n'étant dicté que par la seule information dont dispose son auteur qui prie par avance les concepteurs d'ouvrages non cités de lui pardonner son inexcusable ignorance.

\section{Ouvrages britanniques recensés}

- Vernon BOGDANOR, Devolution in the United Kingdom, Oxford: Oxford University Press, col. OPUS, 1999, 329 p., £ 8.99.

- Arthur AUGHEY, Nationalism, Devolution and the Challenge to the United Kingdom State, Londres: Pluto Press, 2001, 209 p., £ 15.99.

- Peter CATTERAL, Wolfram KAISER, Ulrike WALTON-JORDAN (eds.), Reforming the Constitution, Londres: Frank Cass, 2000, 304 p., £ 17.50.

- Keith SUTHERLAND (ed.), The Rape of the Constitution?, Thoverton: Imprint Academic, 2000, 370 p., $£ 12.95$.

- Anthony SELDON (ed.), The Blair Effect, Londres: Little, Brown and Company, 2001, 661 p., $£ 14.99$.

L'ouvrage de Vernon Bogdanor, exclusivement centré sur la déconcentration des pouvoirs au Royaume-Uni, retrace le cheminement du concept de dévolution qui se définit en termes de transfert de pouvoirs depuis le Home Rule. Partisan du partage des pouvoirs, Bogdanor, citant Dicey en renfort, montre en quoi le Scotland Act de 1998 constitue en fait le premier jalon d'un parcours pouvant mener l'Écosse vers une union de type fédéral avec le reste du Royaume pour peu que les Écossais manifestent par leur 
soutien majoritaire au SNP leur volonté séparatiste. Bogdanor a aussi le mérite de s'attarder sur la "question anglaise ", peu traitée dans les ouvrages recensés, pour en montrer la complexité. Le renforcement du pouvoir des régions anglaises détruirait la structure binaire de l'administration territoriale actuelle, tandis qu'un Parlement d'Angleterre se poserait nécessairement en rival de Westminster. Enfin, la demande identitaire n'est pas telle, sauf dans le Nord-Est, que l'on ne puisse attendre à Londres de la voir prendre un poids politique plus convaincant. Arthur Aughey poursuit une démarche parallèle à celle de Bogdanor. Au cœur de la réflexion de ce spécialiste de science politique, on trouve l'interrogation sur le concept d'identité nationale. Il en décline les variantes historiques dans le contexte britannique et en détaille les différentes colorations selon la culture politique du moment. Les modes d'auto-détermination sont également passés en revue et l'auteur ne néglige pas la « question anglaise » abordée pour l'essentiel sous l'angle de la psycho-anthropologie. Selon Aughey, la solution politique la plus efficace permettant de répondre à la demande de reconnaissance de la réalité anglaise constituerait à favoriser l'élection de maires dans les principales conurbations anglaises, en s'inspirant de l'exemple londonien. Le moindre intérêt de cet ouvrage n'est pas la bibliographie, riche de près de cinq cents entrées, dans laquelle l'auteur puise abondamment pour construire son argumentation.

4 Les trois autres ouvrages de la série britannique sont des ouvrages collectifs aux objectifs sensiblement différents.

5 Celui publié sous la triple autorité de Peter Catteral, Wolfram Kaiser et Ulrike WaltonJordan s'inscrit dans la série des ouvrages patronnés par l'Institute of Contemporary British History. Rassemblant exclusivement des contributions d'universitaires, il se propose de replacer dans un panorama historique l'évolution de trois facteurs jugés essentiels à la compréhension du débat relatif au cadre constitutionnel britannique, à savoir le droit et les institutions, la place des femmes dans le processus d'émancipation politique et social et, enfin, la question des revendications nationales et de l'administration territoriale. L'étude du système des partis et de la réforme électorale y tient une place importante, à côté de thèmes plus attendus tel la réforme de la Chambre des Lords examinée sous l'angle de son rapport à l'institution monarchique qui semble aujourd'hui avoir été évacuée des préoccupations des spécialistes. Plus originale encore, la réflexion autour du concept de citoyenneté qui prend tout son sens dans le débat lancé par l'actuel ministre de l'Intérieur, David Blunkett. On pourra, toutefois, ne pas partager l'opinion de l'auteur, anonyme, de l'avant-propos qui n'hésite pas à considérer le processus de réformes en cours en Grande-Bretagne comme le plus intéressant dans le contexte de la réflexion internationale sur les moyens les plus propres à asseoir la légitimité des démocraties modernes en ces temps de mondialisation.

6 Plus chatoyant et surtout plus éclectique, l'ouvrage édité par Keith Sutherland fait appel à une trentaine de signatures d'universitaires, d'acteurs politiques et de journalistes dont les contributions, parfois contradictoires, sont arbitrées habilement dans l'introduction du responsable éditorial intitulée "Bagehot Revisited». Il est souvent question dans cet ouvrage de révolution, par quoi l'on entend le plus souvent une plus grande démocratisation du système politique. Stimulant et laissant s'exprimer l'engagement des uns et des autres, l'ouvrage couvre l'ensemble des questions qui structurent aujourd'hui la réflexion sur l'identité du Royaume-Uni et le fonctionnement de ses institutions.

7 On tient avec The Blair Effect, dont Anthony Seldon est l'architecte incomparable, la somme irremplaçable qui épuise l'analyse du premier mandat de Tony Blair dans ses 
moindres manifestations. Parmi l'impressionnante équipe réunie par Seldon, on retrouve les noms de Philip Norton, Vernon Bogdanor, Dennis Kavanagh, Ivor Crewe associés à ceux de journalistes réputés, Peter Riddell (Times), Philip Stephen et Robert Taylor ( Financial Times). La politique étrangère, et surtout la question européenne, tiennent une part importante dans ce bilan qui tente d'analyser les raisons de la timidité réformatrice du premier gouvernement Blair, question qui pourrait paraitre paradoxale au vu des restructurations de la machine institutionnelle déjà accomplies. L'étude de ces dernières ne constitue pas, il est vrai, l'essentiel de l'ouvrage qui consacre une place aussi importante à l'analyse du bilan économique, au rôle du Trésor ainsi qu'aux relations avec les milieux d'affaires. Les réactions de l'opinion, la gestion de l'information ne sont pas oubliées dans cet inventaire remarquablement documenté qui dresse un bilan sans concessions d'une action politique appelée à s'inscrire dans la durée.

\section{Ouvrages français recensés}

- Pouvoirs, n 93, "Le Royaume-Uni de Tony Blair", avril 2000, 95 F.

- Témoin, n² 24, “New Labour... Old England ?", mai-juin 2001, 75 F.

- Timothy WHITTON (dir.), Le New Labour. Rupture ou continuité?, Rennes: Presses Universitaires de Rennes, 2000, 282 p., $90 \mathrm{~F}$.

- Emmanuelle AVRIL et Richard DAVIS (dir.), Comprendre la Grande-Bretagne de Tony Blair. Bilan d'une alternance politique, col. Savoirs-mieux, Villeneuve d'Ascq: Presses Universitaires du Septentrion, 136 p., $85 \mathrm{~F}$.

8 Sous le titre "Le Royaume-Uni de Tony Blair", la revue Pouvoirs publiait en avril 2000 un ensemble de textes réunis par Jack Hayward qui offrait un panorama assez diversifié de l'action du gouvernement Blair depuis son accès aux affaires. Parmi les douze contributions qui composent le numéro, trois seulement sont dues à des spécialistes français: Monica Charlot ("Les conservateurs peuvent-ils gagner ?"), Patrick Le Galès ("Dévolution à tous les étages") et Etienne Picard ("Les droits de l'homme et 'l'activisme judiciaire" "). Les « contributeurs » britanniques éclairent, avec méthode, les principaux dossiers institutionnels : la réforme de la Chambre des Lords (Philip Norton), le projet de réforme électorale (David Farrell). La dimension sociale de l'œuvre réformatrice du «nouveau travaillisme» n'est présente que dans l'étude d'Helen Fawcett évaluant la gestion de l'État-Providence. Arnaud Menon, pour sa part, montre qu'en dépit des apparences, le Royaume-Uni a largement réussi son intégration aux institutions européennes. L'ensemble de ces textes s'inscrit dans le prolongement de l'analyse pénétrante que donne David Marquand de l'ancrage idéologique du premier ministre.

9 Revue fondée en 1992 par le club éponyme, Témoin, qui entend nourrir la «réflexion et l'action pour une nouvelle sociale-démocratie», consacrait l'essentiel de sa livraison de maijuin 2001 à un bilan de la première mandature de Tony Blair sous le titre "New Labour... Old England?". On note parmi les auteurs des articles, assez brefs, qui composent le numéro les noms d'acteurs politiques d'hier (Roy Hattersley) et d'aujourd'hui (Jean-Marie Bockel, Pervenche Berès). Un texte de Tony Blair consacré à la politique suivie en matière d'environnement constitue un morceau de choix dans cet ensemble globalement assez critique. Sarah Chapman et Stephen Savage, en particulier, font une analyse sévère de la politique du gouvernement Blair dans le domaine de la lutte contre la délinquance et la criminalité. Plusieurs contributions éclairent la pertinence du concept de «Troisième Voie» par rapport aux choix opérés par d'autres gouvernements - le gouvernement 
allemand en particulier - s'inspirant des thèses social-démocrates. On trouvera dans ce numéro d'utiles remises en perspective historiques développant la filiation idéologique du New Labour et une analyse détaillée du processus de centralisation dans le fonctionnement du parti. En revanche, les grandes réformes institutionnelles sont peu abordées par ces différents textes qui privilégient les aspects sociaux du «Blairisme ».

L'ouvrage publié sous la direction de Timothy Whitton, fruit d'un colloque organisé à Clermont-Ferrand, est intéressant à plus d'un titre. D'abord parce qu'il fait presque exclusivement appel à des universitaires français, même si certains d'entre eux sont d'origine britannique, et, d'autre part, parce qu'il couvre un champ plus vaste que les autres titres figurant dans l'ensemble bibliographique français. L'analyse de la politique économique du gouvernement Blair due à Valérie Auda-André n'a pas son pendant ailleurs, pas plus que l'analyse très fouillée de Whitton relative aux programmes d'aide au retour à l'emploi des jeunes chômeurs. Le fait que près de la moitié des titres des articles soit ponctuée par un point d'interrogation, à l'instar du titre général du volume - Le New Labour : rupture ou continuité ? - illustre bien la difficulté de l'exercice consistant à établir l'inventaire d'une action politique et sociale complexe dont Jean-Paul Révauger s'attache néanmoins à montrer la cohérence. Des rapprochements éclairants peuvent être effectués entre la contribution d'Emmanuelle Avril portant sur le renouveau du militantisme au parti travailliste et le texte de Bernard Moss ("Party Life under New Labour : the Untold Story") dans le numéro de la revue Témoin présenté ci-dessus. On pourra regretter que les réformes institutionnelles ne fassent l'objet que d'un seul éclairage, nécessairement général, mais on se consolera de cette déception en considérant la diversité des thèmes abordés par ce volume de référence.

11 Celui, postérieur, dont Emmanuelle Avril et Richard Davis ont été les maîtres d'œuvre, s'inscrit dans la même lignée des bilans établis par une équipe d'universitaires français comprenant, là encore, quelques Britanniques. Sans prétendre couvrir l'ensemble du champ des politiques, les auteurs abordent les principaux thèmes faisant débat: la politique sécuritaire (Didier Lassalle), la politique sociale (Laurence Maréchal). On note dans cet ouvrage l'intérêt tout particulier accordé aux questions de défense dont traitent deux articles et auxquelles fait largement référence le texte de Pauline Schnapper, déjà mise à contribution dans l'ouvrage de Whitton, axé sur la politique européenne du gouvernement Blair. On portera au crédit des responsables de l'ouvrage d'avoir demandé à Emmanuel Roudaut une étude des rapports de l'administration travailliste avec le monde rural dont il n'existe pas d'équivalent dans l'ensemble des titres recensés. Comparé à l'ouvrage précédent, celui-ci souffre un peu du caractère squelettique de l'appareil critique mais il n'en témoigne pas moins, de façon éloquente, de la permanence de la recherche en civilisation britannique parmi la jeune génération des universitaires français. 


\section{AUTEUR}

\section{JEAN-CLAUDE SERGEANT}

Université Paris III / Maison française d'Oxford 